\title{
A Pragmatic Approach to Conceptual Negotiation Support
}

\author{
Cristóvão Sousa ${ }^{1,2}$, Carla Pereira $^{1,2}$ and António Lucas Soares ${ }^{2,3}$ \\ ${ }^{I}$ CIICESI - ESTGF, Polytechnic Institute of Porto, Rua do Curral, Casa do Curral-Margaride, \\ 4610-156, Felgueiras, Portugal \\ ${ }^{2}$ INESC Porto, Rua Dr. Roberto Frias, s/n 4200, Porto, Portugal \\ ${ }^{3}$ Department of Informatics Engineering, Faculty of Engineering, University of Porto, \\ Rua Dr. Roberto Frias, s/n 4200, Porto, Portugal
}

Keywords: Conceptualisation Process, Ontology Engineering, Conceptual Negotiation, Knowledge Representation.

\begin{abstract}
Collaborative conceptualisation processes are pervasive to most technical and professional activities, but are seldom addressed explicitly due to the lack of theoretical and practical methods and tools to support it. However, it seems not to be a popular research topic in knowledge representation or its sub-areas such as ontology engineering. Our view is that collaboration between stakeholders for specifying an ontology should be addressed at the conceptual, semi-formal level, in order to foster a collective learning of the domain and reaching agreements about its representation. We developed a method to support conceptual integration based in the conceptual blending theory - ColBlend - and implemented it in a collaborative modelling environment. This "conceptual modelling environment - conceptME" supports teams of specialist and facilitators in eliciting conceptual structures with the help of collaborative model editing and terminology services. Conceptual integration and agreements are achieved through the ColBlend method. This paper overviews ColBlend and ConceptME and describes in detail a test case.
\end{abstract}

\section{INTRODUCTION}

Representing knowledge through ontologies usually requires domain experts to commit to some particular formalism, which could derail or at least delay the overall process of achieving a shared representation of concepts and relationships between concepts. Unfortunately, is evident that "While different degrees of formalization have been well investigated and are now found in various ontologybased technologies, the notion of a shared conceptualization is neither well-explored, nor wellunderstood, nor well-supported by most ontology engineering tools" (Staab, 2008). We confirm that current knowledge about the early phases of ontology construction is insufficient to support methods and techniques to support collaborative conceptualisation processes. Trying to address the above identified gap, our research focus on the study and support to collaborative conceptualisation processes. In relation to an individual, a $\mathrm{CP}$ is a set of cognitive activities that has as inputs information and knowledge internally or externally accessible, and as the output an internal or external conceptual representation. Furthermore, a "collaborative conceptualisation process" (CCP) is a $\mathrm{CP}$ that involves more than one individual producing an agreed conceptual representation. It involves social activities that include meaning negotiation and practical management activities for the collaboration (Pereira et al., 2012). CP should account for mechanisms to deal with the inputs for the process once they are crucial to support the conceptualisation tasks. The research reported in this paper addresses the support to the CCB. From a methodological approach developed in previous research (Pereira and Soares, 2008), the so-called ColBlend method, we developed a modelling environment - conceptME - supporting the collaborative creation, editing, discussion and negotiation of conceptual representations (e.g., concept maps, topic maps, UML diagrams). Besides being based on informal knowledge representation notations, more close to the users cognitive representation of a conceptualisation, our approach also provides support to the externalisation of concepts and relations. For this, conceptME provides a suite of terminological services allowing the users to get help from the processing of a previously setup textual corpus about the domain to 
be worked out. More specifically, this paper illustrates the use of the ColBlend method (see section 2), contributing to its improvement and refinement, in particular to what concerns to the implementation of the blend space by means of terminology based techniques assisting users in their conceptualisations activities - more specifically concept elicitation and concept discussion.

\section{SUPPORTING BLEND SPACE: CONCEPTME APPROCAH}

\subsection{Revisiting ColBlend Method}

ColBlend method (see fig. 2) was designed to support a collaborative conceptualisation process, based on conceptual blending theory (CBT) (Fauconnier et al. 1998) (see fig. 1). In practical terms ColBlend aims at supporting the coconstruction of an agreed set of conceptual models, which could be translated into taxonomies, glossaries or ontologies.



Figure 1: CBT.

In (Pereira et al., 2012) ColBlend is detailed. In an overview, the process comprises a set of virtual spaces: a) the input spaces - where each party build models representing their conceptualisation of the domain; b) the blend space - containing the results from the analysis of the input spaces presented for discussion. Moreover, it propose new concepts from a global analysis of the current spaces content and; c) the generic space - which contains the common domain model composed by the all parts of the proposals that were accepted by all and "published" to the this shared space.

ColBlend led to the development of the conceptME (www.concepme.pt). In this paper, we

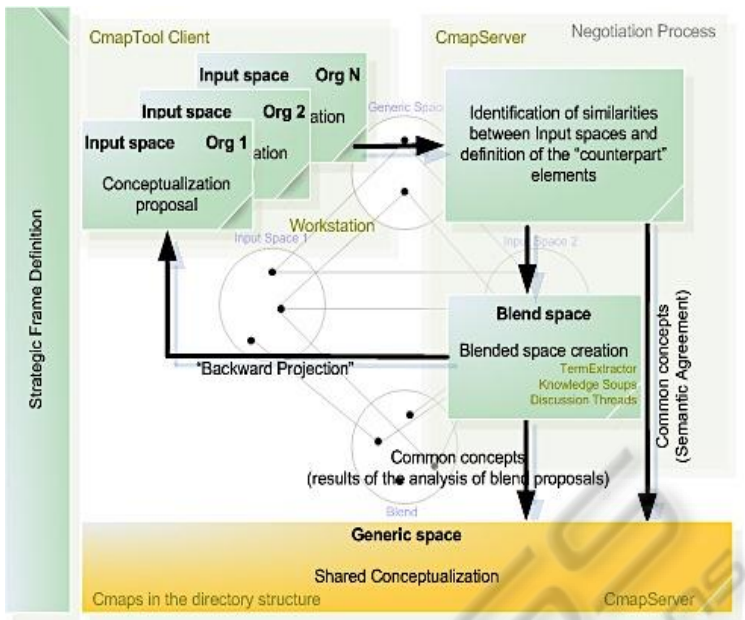

Figure 2: ColBlend method.

present the results of the implementation of the blend space (negotiation support and decisionmaking space) using coordinated corpus-analysis and knowledge representation tasks (the CP main concern). The implementation of blend space is achieved by means of techniques to retrieve immediate contexts of terms, assisting users on their conceptualisations activities - more specifically concept elicitation and concept discussion (designation according to the conceptualisation framework described in (Sousa et al., 2012).

\subsection{ConceptME Approach}

The core of conceptME platform is on supporting collaborative modelling, focusing on graphical knowledge representations and terminological methods, accommodated into a Library of services. The platform is organised as follows (see fig. 3): a) a set of functionalities to manage collaborative modelling projects; b) a collaborative modelling environment, allowing users to build their models individually or editing them collaboratively (either on their own or through available templates), while discussing around concepts; c) a set of terminological services, supported by a domain specific textual corpus, allowing users to associate relevant resources to their projects, performing extraction operations to retrieve candidate terms that can be used in their CP.

At this level, conceptME provides: i) means for corpus organisation and classification; ii) real-time term contexts to detail existing representations; d) a model negotiation baseline to ensure simple negotiation mechanisms, towards a common shared model. This module provides the interface and 


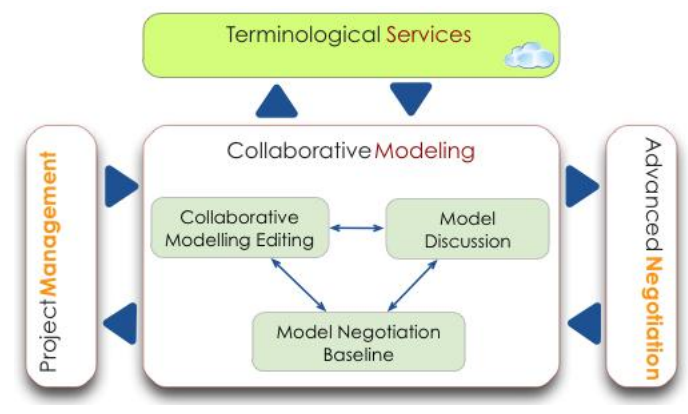

Figure 3: Conceptme high-level architecture.

environment conditions, allowing to connect other advanced negotiation mechanisms, despite of their nature and/or domain.

\subsection{Discussing and Collaborating through Concepts}

In accordance with the principles of the CBT (see fig. 1) and the ColBlend method (see fig. 2), the blend space is implemented by means of three key activities (Fauconnier et al., 1998): composition, completion and elaboration. With the goal to supporting the discussion and collaboration in a collaborative conceptualisation process these activities were designed as follows: The composition activity comprises the identification of the "counterpart" elements (concepts of the input spaces subsumed by concepts of the generic space) of the individual proposals. This calls for specific algorithms to cope with the analysis of common elements between the available models. Available methods in ontology merging perform a one-to-one approach (Doan et al., 2004). Within collaborative settings, where multiple proposals could be available, the question about which pair of models should be analyzed arises. This issue could be mitigated by following a clustering based approach (Araujo et al., 2010). Right before individual proposals appear in the blend space, an intermediate action is triggered whose goal is to provide assistance on defining the order in which composition is performed over the input conceptual structures (ICS) - conceptualisation proposals according to figure 2. Having the elements of each proposal indexed, clustering techniques categorize the ICS into clusters, and then the composition activity will start from the cluster with the highest score. The user could either select the ICS to be analyzed for counterpart elements identification or could accept the platform suggestions. These clustering techniques are available through Solr (http://lucene.apache.org/solr/) by means of carrot clustering engine (http://project.carrot2.org/). As mentioned before, the first step towards blend space is performed by a composition activity. Regarding the conceptME approach, this is accomplished by merging the individual proposals and placing, at the blend space, a model containing the common elements. The other elements are highlighted, becoming potential negotiation targets. Hereafter, comes completion activity comprising a crosschecking corpus-based validation over the elements contained in the ICS. The goal is to provide data to support the decision about the inclusion of the nonshared but non-conflicting elements of the ICS in the blend space. The elaboration activity, by its turn, aims at new concept discovery. This is accomplished by a broader context retrieval, whose focus goes beyond the scope of the elements enclosed in the ICS. Term contexts could be immediately retrieved in order to get clues on possible new concepts or relations related to the current concepts and to the corresponding conceptual representation. Moreover, term contexts could additionally support discussion around a specific concept, justifying its use by showing evidence about term occurrence in corpus or infer on concept semantic metadata (using an RDF triple store) or even highlighting patterns ( $<$ noun $>\langle$ verb $\rangle\langle$ noun $\rangle$ ) within text, indicating the incompleteness of the overall conceptual structure and suggesting new elements to negotiation. The goal is to identify terms (nouns) that co-occur with some other term in the available structures and possible linking phases to connect them. The linking phases are typically verbs or expressions that match the following lexical patterns: i) verb preceded or followed by a preposition or subordinating conjunction; ii) a verb preceded or followed by a coordinating conjunction; iii) a verb preceded or followed by a "TO"; iv) a verb preceded or followed by a determiner and; v) a verb preceded or followed by another verb. These patterns are implemented as xml queries (Xquery/Xpath) and/or regular expressions. A practical experiment resulting of the blend space execution is presented in the next subsection.

\section{ILUSTRATION OF THE APPROACH}

Some experiments were performed exploiting the creation of a corpus about the domain of urban rehabilitation (http://www.h-know.eu/). Three documents selected from the urban rehabilitation corpus were posted (and indexed) on Solr. During 
the conceptualisation process, users added concepts and relationships, either on their own or calling upon extraction services or even through available templates. After the release of a term on canvas, several tasks may follow: a) Provide a definition for the term; b) complete the structure adding another term and a linking phrase between two terms. Context-analysis could help on both. Still in the scope of urban rehabilitation domain, at a certain stage of the $\mathrm{CP}$, the definition of the conceptual structure around the Moisture Control concept was started and a set of input structures were achieved (see fig. $4 a-4 b-4 c$ ).

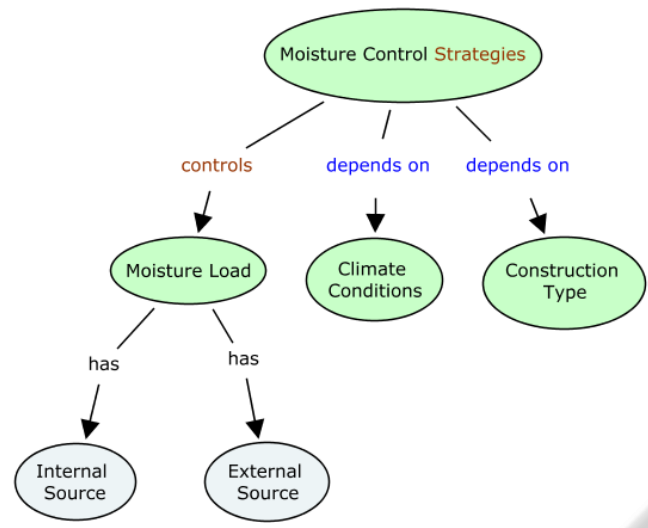

Figure 4a: Input conceptual structure 1.

From the available proposals, Input conceptual structure 1 (fig. 4a) and Input conceptual structure 2 (fig. 4b) are those containing the largest number of counterpart elements, thus, composition will perform over them in first place, unless user has decided differently. In a first iteration, a set of elements was found as being common (Moisture Load, Climate Conditions, Construction Type, Moisture Control and the linking phrase depends on) and was proposed to be merged.

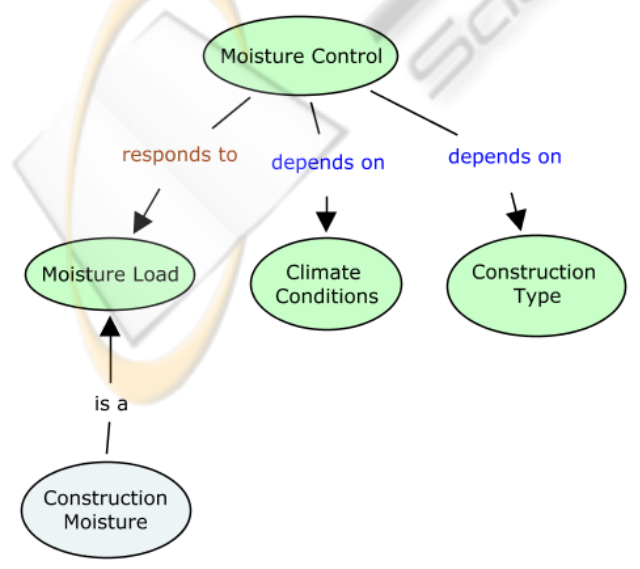

Figure 4b: Input conceptual structure 2.



Figure 4c: Input conceptual structure 3.

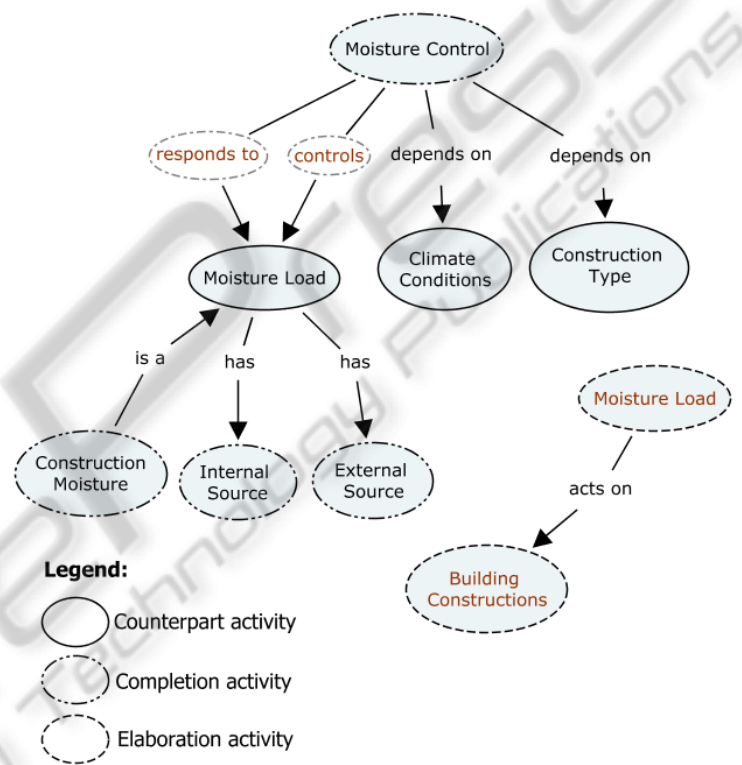

Figure 4d: Blended conceptual structure.

The elements Internal Source, External Source, Construction Moisture and the linking phrases has and is $a$, were proposed to be appended to the resulting structure. The elements in conflict (responds to and controls) were proposed for discussion. Afterwards, completion will perform and the blend space begins to emerge. Grounded on corpus, completion activity checks the composition result performing analysis over the common base information such as: source documents, cooccurrences of the terms in corpus through context retrieving and available definitions, but focusing only on the elements of the ICS. Regarding context analysis information depicted in table 1 , the elements: Construction Moisture, Climate Conditions, Internal and External Sources, remained in the blend space. Moreover, Moisture Control prevails over Moisture Control Strategies, since the first term appears in corpus unlike the second one 
(see table 1). The same happened to responds to and controls linking phrases. Once "responds to" was found as contexts were retrieved, it prevails over "controls" relation. Regarding the structure "Moisture control acts on Building Constructions", it emerges along with the elaboration activity, whilst contexts are processed to reveal specific patterns in the form of <noun $>$ [prefix] $<$ verb $>$ [suffix] $<$ noun $>$ (prefix and suffix are optional and regards to the lexical patterns mentioned earlier in section 3.2). In order to avoid uninteresting results, pattern discovery services considered that, at least a <noun> was already in use in some input structure. Further iterations could disregard such requirement.

Table 1: Excerpt of Moisture control contexts.

Textual corpus Context for "Moisture Control" concept
Effective moisture control has to respond to the exterior as
well as the interior moisture
loads acting on building constructions.
However, good moisture control design depends on a
variety of parameters such
as climate conditions and construction type which changes
from region to region.
Rehabilitation guidelines and moisture
control Some heritage constructions are more vulnerable
to moisture loads than modern buildings.

\section{RELATED WORK}

The work on ontology engineering methodologies has been extensively discussed and compared in (Corcho, 2003), (Fernández-López, 2002), (López et al., 1999) and (Gasevic et al., 2006). We can conclude that the ontology-engineering field has laid a lot of emphasis on the "specification of the conceptualization" as an engineering task. Nevertheless, the early phases of the ontology development life-cycle have been poorly addressed. In particular, the importance of the social processes involved in the formation of a collective conceptualization (e.g., of a domain) has not been recognized. Dealing more directly with collaboration in ontology development (see (Simperl et al., 2006), (Kotis et al., 2006), (Aschoff, 2004), (Zhao, 2005), (de Moor et al., 2006), (Staab et al., 2001), (Sure, 2002), (Gómez-Gauchía et al., 2004), and (Pinto et al., 2004) for a complete account of those approaches), lead to the conclusionthat few research works recognise the importance of supporting the collective construction of a conceptualization. Some particular questions come out from this review: (i) the importance of representational tools and user interfaces for interacting with knowledge representations are generally underestimated; (ii) negotiation and consensus building regarding the conceptualization content has not been a priority either; there are a few proposals that claim to support the process of reaching consensus or agreements, but only one addresses the issue of what conceptual content should be included in the shared conceptualization; (iii) the reutilization of existing ontologies is an obvious requirement; nevertheless, there is not any approach that integrates reutilization with the conceptualization building in a systematic way.

\section{CONCLUSIONS}

This paper addresses the support to collaborative conceptualisation processes, an overlooked research topic in ontology engineering and knowledge representation in general. It showed that conceptual agreements within a group developing initial models for the creation of an ontology, can be improved through the semantic processing of input models based on the Conceptual Blending Theory. Furthermore, the combination of this conceptual integration with basic terminological approaches, providing assistance in concept elicitation and validation, makes this approach quite innovative. The conceptME platform is already available, is free to use and we are planning to realise it as an open source project. Future work will be focused on the running of empirical experiments with two aims: (i) to deepen the knowledge about collaborative conceptualisation processes and (ii) to continue the development of conceptME, specifically in the terminological support and conceptual negotiation techniques. As someone said (Floridi, 2008), "humans are the only semantic engines available", thus we are strongly embracing a socio-semantic perspective in the development of tools for collaborative knowledge representation.

\section{ACKNOWLEDGEMENTS}

This work is funded by the ERDF through the Programme COMPETE and by the Portuguese Government through FCT - Foundation for Science and Technology, project PTDC/EIA-EIA/103779/ 2008 "CogniNET".

\section{REFERENCES}

de Moor, A., Leenheer, P. D., Meersman, R. (2006). 
Dogma-mess: A meaning evolution support system for interorganizational ontology engineering. Proc. of the 14th Inter. Conf. on Conceptual Structures (ICCS 2006), Aalborg, Denmark, July 17-21.

Kotis, K., Vouros, G. (2006). Human-centered ontology engineering: The HCOME methodology. Knowledge and Information Systems, vol. 10 (1), pp. $109-131$.

Staab, S., Studer, R., Schnurr, H., Sure, Y. (2001). Knowledge processes and ontologies. IEEE Intelligent Systems, vol. 16 (1), pp. $26-34$.

Sure, Y. (2002). A tool-supported methodology for ontology-based knowledge management. In ISMIS 2002, Methodologies for Intelligent Systems.

Gómez-Gauchía, H., Díaz-Agudo, B., González-Calero, P. (2004). Towards a pragmatic methodology to build lightweight ontologies: a case study. In Procs. of the IADIS - 2004, Lisboa, Portugal.

Pinto, S., Staab, S., Sure, Y., Tempich, C. (2004). Ontoedit empowering swap: a case study in supporting distributed, loosely-controlled and evolving engineering of ontologies (diligent). In 1st ESWS 2004, Springer.

Aschoff, F. (2004). Knowledge mediation: A procedure for the cooperative construction of domain ontologies. Master's thesis, University of Heidelberg.

Zhao, G. (2005). AKEM: an ontology engineering methodology in FF POIROT. Deliverable 6.8 of FF POIROT project.

Corcho, O., Fernández-López, M., Gómez-Pérez, A. (2003). Methodologies, tools and languages for building ontologies: where is their meeting point?, Data Knowl. Eng., vol. 46 (1), pp. 41 -- 64.

Fernández-López, M., Gómez-Pérez A. (2002) Overview and analysis of methodologies for building ontologies. The Knowledge Engineering Review, vol. 12 (2), pp. $129-156$.

López, M., Gómez-Pérez, A., Sierra, J., Sierra, A. (1999). Building a chemical ontology using methontology and the ontology design environment. IEEE Intelligent Systems, vol. 14 (1), pp. 37 -- 46.

Gasevic, D., Djuric, D., Devedzic, V. (2006). Model Driven Architecture and Ontology Development. Springer-Verlag, Berlin.

Simperl, E., Tempich, C. (2006). Ontology Engineering: A Reality Check. In R. Meersman and Z. Tari and others, The 5th Intenational Conference on Ontologies, DataBases, and Applications of Semantics (ODBASE2006), volume 4275 of Lecture Notes in Computer Science (LNCS), Springer, Montpellier, France.

Staab, S. (2008) On understanding the collaborative construction of conceptualisations. International and Interdisciplinary Conference "Processing TextTechnological Resources" at the Center for Interdisciplinary Research $(\mathrm{ZiF}$ - Zentrum für interdisziplinäre Forschung), Bielefeld University, 1315 March.

Fauconnier, G., Turner, M. (1998). Conceptual Integration Networks. Published in Cognitive Science, vol. 22 (2), pp. $133-187$.
De Araujo, F. F., Lopes, F. L. R. \& Loscio, B. F., 2010. MeMO: A Clustering-based Approach for Merging Multiple Ontologies. In IEEE, pp. 176-180.

Doan, A., Madhavan, J., and Halevy, A., 2004. "Ontology Matching: A Machine Learning Approach". In S. Staab and R. Studer (eds.), Handbook on Ontologies in Information Systems (pp. 385-403). New York: Springer.

Sá, C., Pereira, C., Soares, A. (2010. Supporting collaborative conceptualization tasks through a semantic wiki based platform. OTM 2010 Workshops Lecture Notes in Computer Science, 2010, Volume 6428/2010, 394-403.

Pereira, C., Sousa, C., Soares, A. (2012). (Interoperable) Supporting conceptualisation processes in collaborative networks: a case study in an R\&D project. International Journal of Computer Integrated Manufacturing. Special Issue Paper.

Sousa, C., Soares, A., Pereira, C., Costa, R. (2012). Integrating terminological methods in a framework for collaborative development of semi-formal ontologies". In Proceedings of the colabTKR - collaboration in Terminology and Knowledge, part of the LREC 2012.

Floridi, L., The Semantic Web vs. Web 2.0: a Philosophical Assessment, Episteme, 2009, 6, 25-37.

Pereira, C., \& Soares, A. L. (2008). Ontology Development in Collaborative Networks as a Process of Social Construction of Meaning. In R. Meersman, Z. Tari, \& P. Herrero (Eds.), On the Move to Meaningful Internet Systems: OTM 2008 Workshops, Vol. 5333. Springer Berlin / Heidelberg, pp. 605-614. 